\title{
Towards Tracking Breast Cancer Across Medical Images Using Subject-Specific Biomechanical Models
}

\author{
Vijay Rajagopal ${ }^{1}$, Angela Lee ${ }^{1}$, Jae-Hoon Chung ${ }^{1}$, Ruth Warren ${ }^{2}$, \\ Ralph P. Highnam ${ }^{3}$, Poul M.F. Nielsen ${ }^{1}$, and Martyn P. Nash ${ }^{1}$ \\ ${ }^{1}$ Bioengineering Institute, University of Auckland, NZ \\ v.rajagopal@auckland.ac.nz \\ ${ }^{2}$ Department of Radiology, Addenbrooke's Hospital, Cambridge, UK \\ ${ }^{3}$ Highnam Associates Limited, NZ
}

\begin{abstract}
Breast cancer detection, diagnosis and treatment increasingly involves images of the breast taken with different degrees of breast deformation. We introduce a new biomechanical modelling framework for predicting breast deformation and thus aiding the combination of information derived from the various images. In this paper, we focus on MR images of the breast under different loading conditions, and consider methods to map information between the images.

We generate subject-specific finite element models of the breast by semi-automatically fitting geometrical models to segmented data from breast MR images, and characterizing the subject-specific mechanical properties of the breast tissues. We identified the unloaded reference configuration of the breast by acquiring MR images of the breast under neutral buoyancy (immersed in water). Such imaging is clearly not practical in the clinical setting, however this previously unavailable data provides us with important data with which to validate models of breast biomechanics, and provides a common configuration with which to refer and interpret all breast images.

We demonstrate our modelling framework using a pilot study that was conducted to assess the mechanical performance of a subject-specific homogeneous biomechanical model in predicting deformations of the breast of a volunteer in a prone gravity-loaded configuration. The model captured the gross characteristics of the breast deformation with an RMS error of $4.2 \mathrm{~mm}$ in predicting the skin surface of the gravity-loaded shape, which included tissue displacements of over $20 \mathrm{~mm}$. Internal tissue features identified from the MR images were tracked from the reference state to the prone gravity-loaded configuration with a mean error of $3.7 \mathrm{~mm}$. We consider the modelling assumptions and discuss how the framework could be refined in order to further improve the tissue tracking accuracy.
\end{abstract}

\section{Introduction}

Breast cancer diagnosis and treatment typically involves the use of a number of imaging modalities and views of the breast. X-ray mammography is an effective screening tool for the detection of cancer at an early stage, and involves 
compression of the breast between two plates in the cranio-caudal and mediolateral oblique directions. MR images are taken for further characterization of suspicious lesions found in mammograms. Breast MRI is typically conducted with the subject lying in a prone position with the breasts hanging pendulously within a specialized MR coil.

During breast imaging and treatment procedures, significant shape changes can occur, whereby the internal tissues undergo large deformations between imaging orientations. Consequently, no two images of the breast show exactly the same tissues, thus tracking features between images is a difficult task. To this end, biomechanical models of the breast are being developed in order to constrain non-rigid image registration algorithms in order to provide transformations that are physically admissible 12 .

We present a finite element modelling framework that we are developing to track features between medical images. We demonstrate our modelling approach by describing a pilot study based on MR images of one volunteer. In developing the model, we address two important issues that have not been previously reported: (i) the identification of the reference configuration of the breast when no load is applied (noting that images of the breast are almost always taken under gravity, and possibly compression loading conditions); and (ii) the estimation of subject-specific in-vivo mechanical properties of the breast tissues. Whilst the implications of the latter are somewhat intuitive, the importance of an accurate representation of the unloaded reference configuration of the breast has not been previously discussed. The large nonlinear deformations that the breast experiences can be modelled using the laws of physics, but this type of modelling depends on knowledge of a well-defined stress-free reference configuration. This unloaded state also serves as an ideal shape to which information and features from all images of the breast can be mapped and interpreted, since it is the configuration for which all loading and deformation effects imposed by the imaging procedures are removed. We have recently developed a computational technique to estimate this unloaded reference state [3], but this method remains to be validated for breast imaging in the clinical setting. For present purposes, we have experimentally estimated the reference configuration using neutral buoyancy imaging as explained in Section 2.2

We first describe the methods that were employed to create the subjectspecific biomechanical model for the pilot study. We then assess the performance of the model in predicting breast shape and tissue movement from the neutral buoyancy reference state to the prone gravity-loaded configuration.

\section{Methods}

We use a finite element implementation of large-deformation hyperelasticity theory to model breast biomechanics in our in-house software, CMISS [4. This theory is more suitable to model the nonlinear deformations as opposed to smallstrain linear elasticity techniques, which do not account for the large rotations that typical occur during finite deformations. The reader is directed to [5] and 
[6] for further details on the theory of finite deformations and finite elements, respectively. The following sections outline the methods that were used to create a subject-specific finite element model of the breast under gravity loading conditions.

\subsection{Subject-Specific Breast Geometry}

We use smoothly continuous cubic-Hermite basis functions to represent breast geometry as they capture complex geometries with more realism than lower-order basis functions [7]. While a line represented with a 1D linear basis function has one degree of freedom per node (the $x$-coordinate, for instance), a representation with a $1 \mathrm{D}$ cubic-Hermite basis function consists of 2 degrees of freedom per node: the coordinate value and its arc-length derivative. The additional derivative information at the nodes implicitly enforces continuity of the spatial gradients at the interfaces between elements, thus requiring fewer elements to represent smoothly continuous anatomical surfaces compared to linear basis functions. Since cubic-Hermite functions use a greater number of degrees of freedom per node than linear basis functions, it follows that the use of fewer elements does not necessarily correspond to fewer geometric degrees of freedom. Indeed, for equal numbers of total geometric degrees of freedom, higher order basis functions are favoured because they provide better rates of solution convergence for finite elasticity problems [6].

Subject-specific finite element models are generated using a semi-automatic method that efficiently parameterizes the skin and muscle surface contours using geometric data that have been segmented from MR images (Fig. 1) 8]. The method uses a nonlinear least-squares approach to fit surfaces of volume elements to the segmented data sets [7].
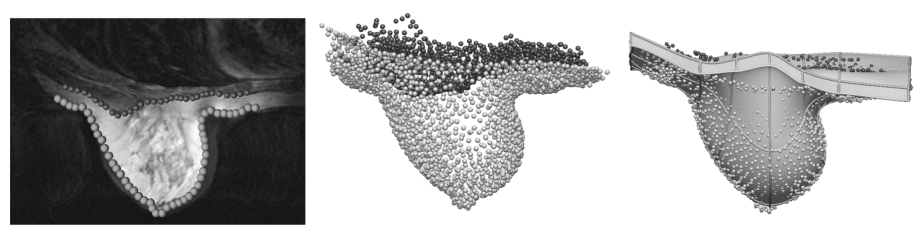

Fig. 1. Process of creating a subject-specific breast model. Left: Segmentation of tissue boundaries from an MR image. Grey points represent the skin surface, and black points represent muscle surface. Middle: Dataset of skin (grey points), and muscle (black points) after segmenting an entire MR image set. Right: Finite element model fitted to skin and muscle surface data.

This customization technique has been applied to MR datasets from six subjects with markedly different breast shapes. We found that geometric finite element models consisting of 24 tri-cubic Hermite elements (with 70 nodes and 1680 total geometric degrees of freedom) fitted the models with an average overall RMS error of $1.5 \mathrm{~mm}$ in representing the skin and muscle surfaces (see Fig. 2). For the mechanics study presented here, the breast was assumed to be 


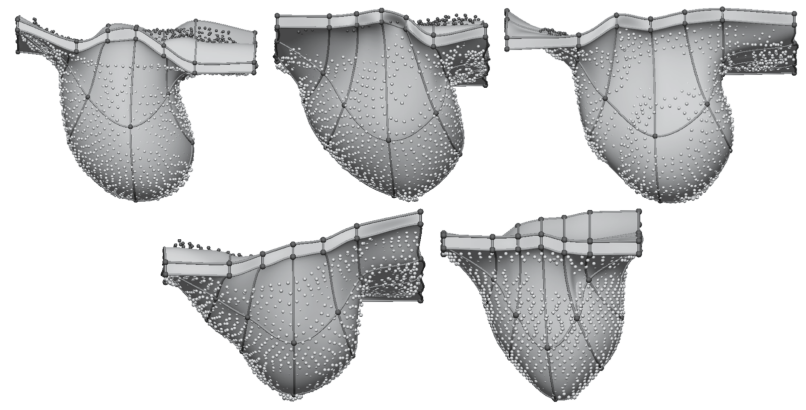

Fig. 2. Finite element model (surfaces) fitted to the skin surface data sets (spots) of 5 subjects lying prone in an MR scanner. The fitted model of subject 1 is shown in Fig. 1

homogeneous after [2, thus the geometrical model did not need to account for any structural differences between the internal tissues.

\subsection{Neutral Buoyancy Imaging and the Unloaded Configuration}

Previous models have typically used the prone gravity-loaded configuration of the breast as the mechanical reference state. However, the effects of gravity loading mean that this is not the true stress-free reference configuration of the breast, hence this assumption may lead to large errors in mechanics predictions. In order to overcome this issue, we obtained MR images of the volunteer's breast when immersed in water and assumed that breast tissue density is close to that of water (see Fig. (4). Thus, we assume that neutral buoyancy offsets the effects of gravity, and more closely represents the unloaded configuration of the breast. These images are the first to capture the unloaded shape of the breast and will be used in future studies to investigate and quantify the importance of the representation of the reference configuration on the accuracy of breast mechanics modelling. For present purposes, neutral buoyancy images were used to create a finite element model of the volunteer's breast in the unloaded configuration. This neutral buoyancy model was used as the reference state for a mechanics simulation of the prone gravity-loaded configuration.

\subsection{Loading and Boundary Conditions}

Gravity loading was applied as a body force to the neutral buoyancy model. For the purposes of this study, it was assumed that the breast tissues and muscle were firmly attached to the rib cage, thus we applied fixed-displacment boundary conditions at the posterior surface of the model. Previous modelling studies have applied displacement boundary conditions to nodes across the entire skin surface to ensure that the outer shape of the breast in the deformed configuration was matched [12]. In contrast, the simulations in the present study are driven by the gravity loading condition alone and thus provide a more robust way of assessing the ability of the model to predict breast deformations. 


\subsection{Subject-Specific Mechanical Properties}

The breast was assumed to be incompressible, homogeneous and isotropic as characterized by the neo-Hookean constitutive equation $W=c_{1}\left(I_{1}-3\right)$, where $I_{1}$ is the first principal invariant of the Lagrangian strain and $c_{1}$ is the material parameter that must be determined for the tissues 5 . Previous models have also used the neo-Hookean relationship, and they have typically used experimental data from the literature in order to characterize the mechanical behavior of breast tissues 112. However, it is well known from experiments that breast tissue mechanical properties vary significantly between subjects 9 . Therefore, it is likely to be important to use a constitutive relationship that is customized to each individual. We used our existing material parameter optimization techniques $3\left[8\right.$ to estimate the value of $c_{1}$ using the model of the neutral buoyancy shape together with the segmented data from the images of the prone gravity-loaded configuration.

\section{Results}

The geometric finite element model of the neutrally buoyant breast had an RMS error of $0.78 \mathrm{~mm}$ for fitting the skin surface and $1.2 \mathrm{~mm}$ for fitting the muscle surface of the volunteer's breast. A displacement solution mesh convergence analysis was performed for the mechanics model by tracking eight material points located throughout the model and recording their displacements for successive refinements. The Euclidian displacements that each material point underwent during the deformation was calculated for each mesh resolution. The RMS errors between successive refinements were then recorded (see Fig 3) and the mesh

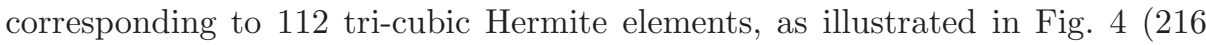
nodes; 5184 geometric degrees of freedom), was chosen as the most appropriate resolution for reliable model predictions. The material parameter optimization technique estimated a value of $c_{1}=0.08 \mathrm{kPa}$ for this model.

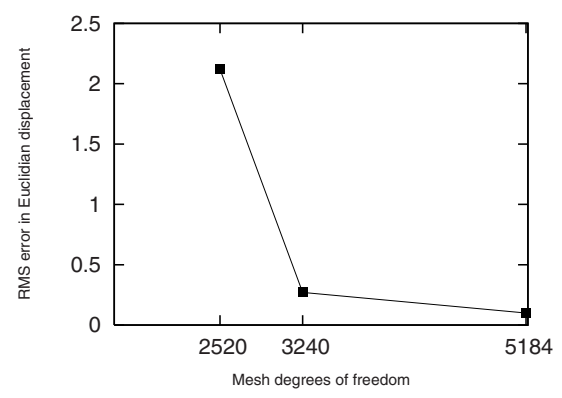

Fig. 3. Convergence of RMS error in Euclidean displacements of selected material points inside the fitted model with increasing mesh resolution 


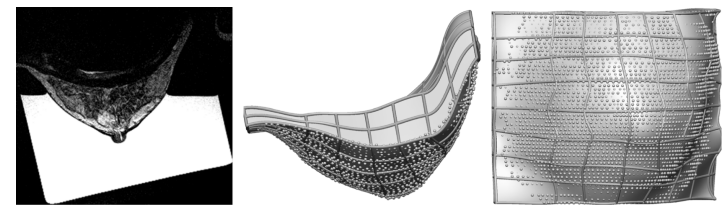

Fig. 4. MR image (Left) and refined finite element model (Right) of the right breast of a volunteer under neutral buoyancy. The bright region in the MR image represents the water bath.

\subsection{Predicting the Prone Gravity-Loaded Configuration}

Figure 5 illustrates the model's ability to predict the deformation of the breast in the prone gravity-loaded configuration. It shows that the homogeneous model provides a good match to the prone gravity-loaded configuration. The segmented surface data from the prone gravity-loaded shape was predicted with an RMS error of $4.2 \mathrm{~mm}$. Four internal tissue regions were segmented from the neutral buoyancy images and tracked using the model to the prone gravity-loaded configuration. These segmented regions were not represented using finite elements, but were tracked as a set of embedded material points in the homogeneous model. Figure 5 illustrates the tracking process and Table 1 quantifies the accuracy with which the individual image features were tracked. On average, the homogeneous model tracked internal tissue deformations with an error of $3.7 \mathrm{~mm}$.

Table 1. Total displacements of the four tissue regions tracked from the neutral buoyancy to the gravity-loaded configuration and the corresponding Euclidean errors between centroids of actual and predicted locations for the gravity-loaded configuration

\begin{tabular}{|c|c|c|c|c|}
\hline Tissue region & 1 & 2 & 3 & 4 \\
\hline Displacement $(\mathrm{mm})$ & 5.06 & 20.47 & 19.0 & 21.7 \\
\hline Euclidean Error $(\mathrm{mm})$ & 1.56 & 4.82 & 4.51 & 3.97 \\
\hline
\end{tabular}

\section{Discussion and Conclusions}

This pilot study has shown that a homogeneous, subject-specific model is able to predict breast deformation from a neutral buoyancy state to a prone gravityloaded configuration within a clinically acceptable localization accuracy (under $5 \mathrm{~mm}$, after [2]). The gross surface characteristics are also reproduced by the model, tracking skin surface deformations with an RMS error of $4.2 \mathrm{~mm}$. It is likely that incorporation of more structural detail, such as heterogeneities in breast tissue properties, will improve model predictions. As identified previously, a realistic representation of the boundary conditions at the chest wall may also improve accuracy [1/2]. Note however, that previous studies have enforced the surface of the model to match the skin surface configuration on the image using displacement boundary conditions. Our work predicts the entire deformation 


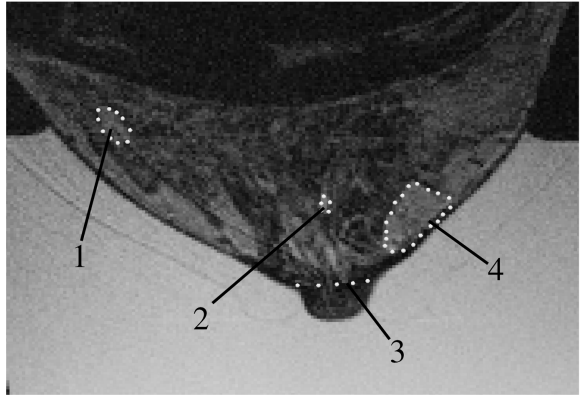

(a)

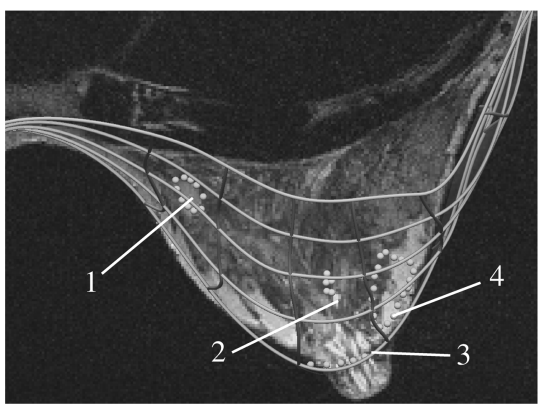

(c)

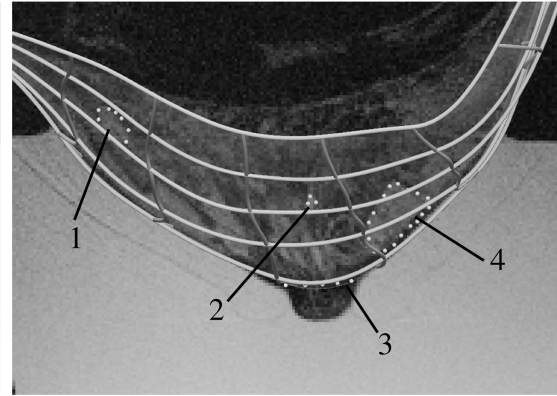

(b)

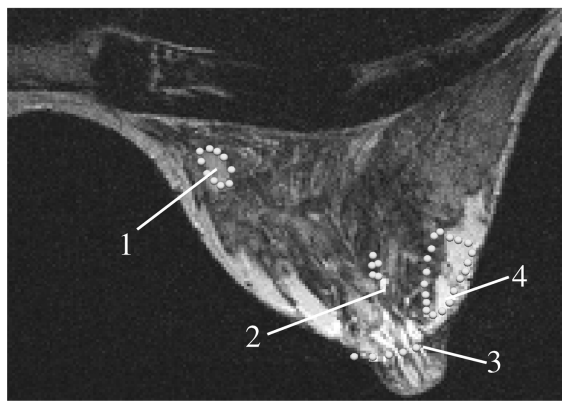

(d)

Fig. 5. Tracking surface and internal tissues from the neutral buoyancy reference configuration to the prone gravity-loaded deformed state. (a): Neutral buoyancy MR image with tissue features circled by white points and numbered sequentially. (b): Fitted finite element model of the unloaded state superimposed on the neutral buoyancy MR image and embedded with the same tissue features. (c): Predicted finite element model of the prone gravity-loaded configuration superimposed on prone gravity-loaded breast MR image. White points show predicted locations of the four tracked tissue features and the labelled points indicate actual locations of the features in the image. (d): Prone gravity-loaded breast MR image superimposed with the predicted locations of tracked tissue features in white, together with actual locations identified and labelled in white. See Table 1 for quantitative comparisons of tissue feature localization.

based only on the loading conditions, mechanical properties and fixed boundaries, thus providing a more robust validation of the model's predictability.

The framework presented in this paper incorporates the use of subject-specific mechanical properties and anatomically realistic geometry in modelling breast deformations. By acquiring neutral buoyancy images, we now have sufficient data to systematically assess the importance of each of the parameters (reference configuration, material properties, loading conditions and boundary conditions) of the biomechanical model. Results of these studies will help inform the modelling regarding the incorporation of biological detail that is necessary to perform 
reliable breast deformation predictions without compromising the speed of computations, which will be important for applications in the clinical setting such as image registration and biopsy procedures.

\section{References}

1. Tanner, C., Schnabel, J., Hill, D., Hawkes, D., Leach, M., Hose, D.: Factors influencing the accuracy of biomechanical breast models. Medical Physics Journal 33(6), 1758-1769 (2006)

2. Ruiter, N., Stotzka, R.: Model-based registration of x-ray mammograms and MR images of the female breast. IEEE Transactions on Nuclear Science 53(1), 204-211 (2006)

3. Rajagopal, V., Chung, J., Bullivant, D., Nielsen, P., Nash, M.: Finite elasticity: Determining the reference state from a loaded configuration. International Journal of Numerical Methods in Engineering (in press, 2007)

4. CMISS. software program (2007), http://www.cmiss.org

5. Fung, Y.: A First Course in Continuum Mechanics. Prentice-Hall, Englewood Cliffs (1977)

6. Zienkiewicz, O., Taylor, R.: The Finite Element Method: The Basis, 5th edn. vol. 1. Butterworth-Heinemann (2000)

7. Bradley, C., Pullan, A., Hunter, P.: Geometric modelling of the human torso using cubic Hermite elements. Annals of Biomedical Engineering 25(1), 96-111 (1997)

8. Rajagopal, V.: Modelling Breast Tissue Mechanics Under Gravity Loading. PhD thesis, University of Auckland (2007)

9. Sarvazyan, A., Skovoroda, A., Emelianov, S., Fowlkes, J., Pipe, J., Adler, R., Buxton, R., Carson, P.: Biophysical bases of elasticity imaging. Acoustic Imaging 21, 223-240 (1995) 